The Egyptian International Journal of Engineering Sciences \& Technology, Vol 9, No 1 (2005)

\title{
AUTOMATIC HUMAN FACE DETECTION IN COLOR IMAGES
} i.f elnahry

\begin{abstract}
Face detection is fundamental for several importaiil applications, such as, face recognition, human- computer interaction, and face image data base management. This paper addresses a technique to automatically detect the person's face from a given image that consists of a head and shoulders. The method involves a fast, simple and yet robust algorithm that classify image pixels into skin color and non- skin color using Days decision rule. Then, based on the spatial arrangement of the detected skin color pixels, the algorithm can be generate face candidates. The proposed technique constructs eye and mouth for verifying each face candidate. Experimental results demonstrate that this teclmique can achieve successful face detection over a wide range of facial variations in color, positions, scale and orientation.
\end{abstract}

